\title{
ON SUM-FREE SUBSEQUENCES
}

\author{
BY
}

\author{
S. L. G. CHOI, J. KOMLOS AND E. SZEMEREDI
}

ABSTRACT. A subsequence of a sequence of $n$ distinct integers is said to be sum-free if no integer in it is the sum of distinct integers in it. Let $f(n)$ denote the largest quantity so that every sequence of $n$ distinct integers has a sum-free subsequence consisting of $f(n)$ integers. In this paper we strengthen previous results by Erdös, Choi and Cantor by proving

$$
(n \log n / \log \log n)^{1 / 2} \ll f(n) \ll n(\log n)^{-1} \text {. }
$$

1. Given a sequence of $n$ distinct integers, a subsequence is said to be sumfree if no integer in it is the sum of distinct integers of the same subsequence. Let $f(n)$ denote the largest quantity so that every sequence of $n$ distinct integers has a sum-free subsequence consisting of $f(n)$ integers. Some lower and upper estimates for $f(n)$ are known (see [1] - [4]). In this paper we strengthen these previous bounds by proving

\section{THEOREM. We have}

$$
(n \log n / \log \log n)^{1 / 2} \ll f(n) \ll n(\log n)^{-1} .
$$

2. In this section we establish the upper estimate in (1.1). First we prove the following Lemma which is perhaps of some independent interest.

Lemma. Suppose $a_{1}<\cdots<a_{t_{1}}$ is a sequence so that the number of distinct sums $a_{i}+a_{j}$ is $\leqslant t_{1}^{2-c}$. Then the number of integers $x$ so that $a_{i}+a_{j}=$ $x$ has at least $t_{1}^{\alpha}$ solutions, where $\alpha<c$, is at least $2 t+O\left(t^{1-(c-\alpha) / 2}\right)$.

Proof. Denote $t_{1}^{1-(c-\alpha) / 2}$ by $M$. Let $S_{1}$ consist of the first $M$ integers in the sequence and $S_{2}$ the last $M$ integers in the sequence. Let $S_{3}$ denote the rest of the sequence. We shall prove that in $S_{2}+S_{3}$, the number $N$ of integers $x$ so that $a_{i}+a_{j}=x$ has at least $t_{1}^{\alpha}$ solutions is at least $t_{1}+O(M)$, and that a similar conclusion holds for $S_{1}+S_{3}$. As the treatment of $S_{1}+S_{3}$ and that of $S_{2}+S_{3}$ are almost identical, it suffices to establish the assertion for $S_{2}+S_{3}$. On noting that for each integer $x$ in $S_{2}+S_{3}$ there are at most $\left|S_{2}\right|=M$ solutions for

Received by the editors August 13, 1974.

AMS (MOS) subject classifications (1970). Primary $10 \mathrm{L10.}$

Key words and phrases. Sum-free, subsequence, integers.

Copyright $\odot$ 1975, American Mathematical Socicty 
$x=a_{i}+a_{j}, a_{i} \in S_{2}, a_{j} \in S_{3}$, and recalling that the number of distinct integers in $S_{2}+S_{3}$ is at most $t_{1}^{2-c}$, we obtain

$$
\begin{aligned}
N & \geqslant\left(\left|S_{3}\right|\left|S_{2}\right|-t_{1}^{2-c} t_{1}^{\alpha}\right) /\left|S_{2}\right|=t_{1}-2 M-t_{1}^{2-c+\alpha} M^{-1} \\
& =t_{1}-3 M=t_{1}-3 t_{1}^{1-(c-\alpha) / 2} .
\end{aligned}
$$

This completes the proof of the Lemma.

We now proceed to establish the upper estimate in (1).

We put

$$
t=\left[n((\log n) / 3)^{-1}\right]
$$

and determine $s$ so that

$$
t(s+1) \leqslant n<t(s+2) .
$$

We define a set $A$ of $n$ integers as follows: $\left({ }^{1}\right)$

For each $i=0,1, \ldots, s$, let

$$
A_{i}=2^{i}[t, 2 t)
$$

Let $A_{s+1}$ consist of any $n-t(s+1)$ integers distinct from those in $A_{0}, \ldots, A_{s}$. Finally, put $A=A_{0} \cup \cdots \cup A_{s+1}$. Suppose $B$ is a sum-free set from $A$. We shall establish the theorem by showing that

$$
|B| \ll n(\log n)^{-1} \text {. }
$$

For each $i=0, \ldots, s$, we denote by $B_{i}$ the intersection of $A_{i}$ and $B$. Let $B_{h}$ be the first set with at least $t^{9 / 10}=t^{1 / 2+2 / 5}$ integers. For $i=h, h+1, \ldots$ we define $B_{i}^{*}, B_{i+1}^{\prime}$ inductively as follows:

Let $B_{h}^{*}$ be a subset of $B_{h}$ consisting of at least $\left|B_{h}\right| / 2$ integers of the same parity. (Note that the pairwise sums of integers in $B_{h}^{*}$ clearly lie in $A_{h+1}$.) Let $B_{h+1}^{\prime}$ consist of those integers $x$ so that $a_{i}+a_{j}=x$ has at least $t^{2 / 5}$ solutions in $a_{i}, a_{j} \in B_{h}^{*}$.

Suppose $B_{k}^{*}, B_{k+1}^{\prime}$ have been defined. We let $B_{k+1}^{*}$ be a subset of $B_{k+1}^{\prime}$ $\cup B_{k+1}$ consisting of at least $\left(\left|B_{k+1}^{\prime} \cup B_{k+1}\right|\right) / 2$ integers of the same parity. Finally we let $B_{k+2}^{\prime}$ denote the set of integers $x$ so that $a_{i}+a_{j}=x$ has at least $t^{2 / 5}$ solutions in $a_{i}, a_{j} \in B_{k+1}^{*}$.

We shall now deduce (2.4) on the assumption that

(1) For real numbers $\alpha>\beta$ and integer $y$, we use $y[\alpha, \beta)$ to denote the set of integers $y m$, where $m$ runs through all integers in $[\alpha, \beta)$. 


$$
B_{i}^{\prime} \cap B_{i}=\varnothing, \quad i=h+1, h+2, \ldots,
$$

which we shall prove later.

We shall apply the Lemma with $a_{1}<\cdots<a_{t_{1}}$ consisting of the integers in $B_{h}^{*}$ so that

$$
t_{1}=\left|B_{h}^{*}\right|=\left|B_{h}\right| / 2 \geqslant t^{9 / 10} / 2 .
$$

Therefore, since $B_{h}^{*} \subset B_{h} \subset 2^{h}[t, 2 t)$ and since all the integers in $B_{h}^{*}$ are of the same parity, we may conclude that the number of distinct sums $a_{i}+a_{j}$ is $\leqslant t \leqslant$ $\left(2\left|B_{h}^{*}\right|\right)^{10 / 9} \leqslant\left|B_{h}^{* P^{33 / 20}}=\right| B_{h}^{* 2-17 / 20}$ provided $t$ is sufficiently large which we may clearly assume. Next, again assuming $t$ sufficiently large, we have $\mid B_{h}^{* \rho / 20} \geqslant$ $t^{2 / 5}$. Thus we may apply the Lemma with $c=17 / 20$ and $\alpha=9 / 20$ to obtain

$$
\left|B_{h+1}^{\prime}\right| \geqslant 2\left|B_{h}^{*}\right|+O\left(\left|B_{h}^{*}\right|^{1-1 / 2((17-9) / 20)}\right)=\left|B_{h}\right|+O\left(t^{4 / 5}\right) .
$$

Similarly, $\left|B_{n+2}^{\prime}\right| \geqslant 2\left|B_{n+1}^{*}\right|+O\left(\left|B_{n+1}^{*}\right|^{4 / 5}\right)$, which, since $B_{n+1}^{\prime} \cup B_{n+1}$ is a disjoint union on using (2.5), is $\geqslant\left|B_{h+1}^{\prime}\right|+\left|B_{h+1}\right|+O\left(t^{4 / 5}\right)$. In general, we have

$$
\left|B_{k}^{\prime}\right| \geqslant\left|B_{k-1}^{\prime}\right|+\left|B_{k-1}\right|+O\left(t^{4 / 5}\right) .
$$

Thus

Hence

$$
t \geqslant\left|B_{s+1}^{\prime}\right| \geqslant \sum_{i=h}^{s-1}\left|B_{i}\right|+O\left(t^{4 / 5} s\right)
$$

$$
|B|=\sum_{i=0}^{s+1}\left|B_{i}\right| \leqslant t^{9 / 10} s+O\left(t^{4 / 5} s\right)+2 t=2 t+o(t),
$$

and in view of (2.1) and (2.2) this proves (2.4).

It remains to show (2.5). We shall actually establish the stronger result that for each $i=1, \ldots, s-h$, any $2^{s-i}$ distinct integers in $B_{h+i}^{\prime}$ have distinct representations; i.e. if $x_{k}\left(k=1, \ldots, 2^{s-i}\right)$ are any $2^{s-i}$ distinct integers in $B_{h+i}^{\prime}$, then there exist $\Sigma_{k=1}^{2 s-i} N_{k}$ distinct integers $x_{k, j}\left(k=1, \ldots, 2^{s-i} ; j=1, \ldots\right.$, $N_{k}$ ) belonging to $B$ such that $x_{k}=x_{k, 1}+\cdots+x_{k, N_{k}}$.

For $i=1$ this is clearly true since each integer in $B_{h+1}^{\prime}$ is the sum of at least $t^{2 / 5}$ pairs of integers in $B_{h}^{*}$. (Note that $t^{2 / 5} \geqslant\left(n \log ^{-1} n\right)^{2 / 5}>n^{1 / 2}>2^{s}$ in view of (2.1) and (2.2).) Now suppose the above result has been established for $i \leqslant k-1$. We shall prove that it holds for $k$ also. So accordingly let $x_{1}, \ldots, x_{Q}$, be $Q=2^{s-k}$ distinct integers in $B_{h+k}^{\prime}$. Since each $x_{j}(j=1, \ldots, Q)$ can be expressed as the sum of two integers in $B_{h+k-1}^{*}$ in at least $t^{2 / 5}$ ways, we may thus have 


$$
\begin{gathered}
x_{1}=y_{1}+z_{1} \\
\cdot \\
\cdot \\
x_{Q}=y_{Q}+z_{Q},
\end{gathered}
$$

where $y_{1}, z_{1}, \ldots, y_{Q}, z_{Q}$ are $2 Q=2^{s-(k-1)}$ distinct integers in $B_{h+k-1}^{*}$. Some of these may of course be in $B_{n+k-1}$, but at any rate at most $2^{s-(k-1)}$ are in $B_{h+k-1}^{\prime}$. Now the induction hypothesis yields the result. This completes the proof of (2.5) and with it that of (2.4).

3. In this section we shall establish the lower estimate in (1.1). Suppose

$$
a_{1}<\ldots<a_{n}
$$

are any $n$ positive integers. We shall proceed to select a sum-free subsequence with $\geqslant \sqrt{n} w(n)$ elements, where $\left({ }^{2}\right)$

$$
w=w(n)=(1 / 40) \sqrt{\log n(\log \log n)^{-1}} .
$$

The semiopen intervals $B_{s}=\left[2^{s}, 2^{s+1}\right)$ are called blocks, and the neighbourhood of the block $B_{s}$ consists of all blocks $B_{j}$ for which $|j-s|<\log \log n$.

On observing that any set, either consisting of integers all from the same block or consisting of integers each from a different nonadjacent block, forms a sum-free set, we may assume that we have $k$ blocks, where

$$
\sqrt{n}(2 w)^{-1} \leqslant k \leqslant \sqrt{n w}
$$

each consisting of at least $\sqrt{n}(2 w)^{-1}$ and at most $\sqrt{n} w$ of the numbers in (3.1). Put

$$
l=\left[\sqrt{n}(2 w \log \log n)^{-1}\right] ; \quad t+1=[(\log n) / 5] .
$$

We can choose $l$ of these blocks say $B_{i_{1}}, \ldots, B_{i_{l}}$ such that

$$
i_{j+1}-i_{j} \geqslant \log \log n, \quad j=1, \ldots, l-1 \text {. }
$$

By a theorem of Chvátal and Komlós [5, Theorem 4], in each block $B_{i_{j}}$ there are at least $t+1$ elements, $b_{1}<\cdots<b_{t+1}$ such that the $t$ consecutive differences $b_{2}-b_{1}, b_{3}-b_{2}, \ldots, b_{t+1}-b_{t}$ form an increasing or a decreasing sequence. Assume first that we have

$$
M=[l / 2]
$$

(2) The functions $\log n, \log \log n$ will always be taken to the base 2 . 
blocks $B_{i_{j}}\left(j=j_{1}, \ldots, j_{\mathrm{M}}\right)$ so that there exist in $B_{i_{j}} t+1$ numbers $b_{j, 1}, \ldots$, $b_{j, t+1}$ such that the $t$ pairs $b_{j, 2}, b_{j, 1} ; b_{j, 3}, b_{j, 2} ; \cdots ; b_{j, t+1}, b_{j, t}$ give rise to $t$ differences $b_{j, 2}-b_{j, 1} ; b_{j, 3}-b_{j, 2} ; \cdots ; b_{j, t+1}-b_{j, t}$ forming an increasing sequence.

We shall consider these $t M$ pairs of integers in these $M$ blocks and prove

Proposition $P$. There exist $Q \geqslant t M / 60$ pairs of integers contained in a number of blocks such that the neighbourhood of each block cannot contain the difference of a pair of integers in another block.

We shall postpone the proof of Proposition P till later but shall first indicate how it leads to the existence of a sum-free subsequence of (3.1) with $\geqslant \sqrt{n} w(n)$ elements, where $w(n)$ satisfies (3.2).

We let $c_{1}, \ldots, c_{Q}$ be the greater numbers of the $Q$ pairs of integers whose existence is asserted in Proposition P. We shall show that it is sum-free; we note that $Q \geqslant t M / 60$ indeed implies $Q \geqslant w \sqrt{n}$ in view of (3.2), (3.4) and (3.6).

Accordingly let $e_{1}<\cdots<e_{k}$ be $k$ of the integers $c_{1}, \ldots, c_{Q}$, so that $e_{k}=e_{k-1}+\cdots+e_{1}$. It is clear that $e_{k}$ and $e_{k-1}$ must belong to the same block. If $e_{k}=b_{j, i}$ and $d_{j, i}=b_{j, i}-b_{j, i-1}$ then

$$
d_{j, i} \leqslant e_{k}-e_{k-1} \leqslant t d_{j, i}
$$

the right-hand inequality arising from the fact that $d_{j, i}, i=1, \ldots, t$, form an increasing sequence. On the other hand,

$$
e_{k-2} \leqslant e_{1}+\cdots+e_{k-2}<(\log n) e_{k-2} / 4
$$

since $t \leqslant(\log n) / 5$ by (3.5), each block contains at most $t$ integers of $c_{1}, \ldots, c_{Q}$ and (3.5) holds. Thus we obtain from (3.7), (3.8)

$$
5 e_{k-2}(\log n)^{-1}<d_{j, i}<\left(e_{k-2} \log n\right) / 4
$$

and this means that $d_{j, i}$ lies in the neighbourhood of the block containing $\boldsymbol{e}_{\boldsymbol{k - 2}}$. Since $d_{j, i}=b_{j, i}-b_{j, i-1}$ and $e_{k}=b_{j, i}$ we have the existence of a pair of integers, the greater of which is $e_{k}$, such that their difference is in the neighbourhood of the block containing $e_{k-2}$. As it is clear that the block containing $e_{k}$ is different from the one containing $e_{k-2}$, we have obtained a contradiction to the assertion in Proposition $\mathbf{P}$.

We remark that in the case where there are $M$ blocks $B_{i_{j}}\left(j=j_{1}, \ldots, j_{M}\right)$ each with $t+1$ integers giving rise to $t$ consecutive differences forming a decreasing sequence, the proof regarding the existence of $Q$ pairs satisfying Proposition $\mathrm{P}$ will be the same and the only modification occurs in the deduction from Proposition 
$P$ where we now take the smaller integers of the $Q$ pairs and obtain in place of (3.7) the inequality

$$
d_{j, i+1} \leqslant e_{k}-e_{k-1} \leqslant t d_{j, i+1}, \text { where } e_{k-1}=b_{j, i}
$$

It remains to prove Proposition P. We first introduce the following definition of a bad block.

A block is said to be bad if one of the following holds:

(i) it contains $\geqslant t / 10$ pairs of integers and its neighbourhood contains their differences;

(ii) its neighbourhood contains the differences of $11 t / 10$ pairs of integers.

It is clear that we may assume there are $<M / 2$ bad blocks, since if (i) holds for $\geqslant[M / 4]$ blocks we already have Proposition $P$ by simply taking the $[M / 4] t$ pairs in these blocks, and if (ii) holds for [M/4] blocks then we obtain $(11 / 10)[M / 4]$ pairs of integers whose differences lie in their neighbourhood, so that after discarding those pairs which actually lie in these blocks we obtain $\geqslant$ $(t / 10)[M / 4]>M t / 60$ pairs of integers satisfying the assertion in the proposition.

Hence we may take

$$
M^{\prime}=[M / 2]+1
$$

blocks which are not bad. We may further assume that there are at most $M^{\prime} t / 20$ pairs in these $M^{\prime}$ blocks with differences lying outside the neighbourhoods of these $M^{\prime}$ blocks. Thus we may assume there are $M^{\prime}$ blocks which are not bad and contain altogether at least $19 M^{\prime} t / 20$ pairs of integers whose differences actually lie in the neighbourhoods of these $M^{\prime}$ blocks. On this assumption we shall obtain Proposition $\mathrm{P}$ by proving that for each natural number $j \leqslant\left(M^{\prime} / 13\right)+1$ there exist [jt/2] pairs of integers and $j$ blocks $B_{p_{1}}, \ldots, B_{p_{j}}$ such that all these pairs lie outside $B_{p_{1}}, \ldots, B_{p_{j}}$ but their differences lie in the neighbourhoods of these blocks. We shall prove this by induction.

Since there are $\geqslant 19 M^{\prime} t / 20$ pairs of integers whose differences lie in the neighbourhoods of $M^{\prime}$ blocks, there exists one block, say $B_{p_{1}}$, whose neighbourhood contains the differences of $\geqslant 19 t / 20$ pairs of integers. Since $B_{p_{1}}$ is not bad, it can contain at most $t / 10$ of these pairs. Thus we may choose [t/2] pairs of integers not lying. in $B_{p_{1}}$ but with differences lying in the neighbourhood of $B_{p_{1}}$. This establishes the assertion for $j=1$. Suppose now $1 \leqslant j \leqslant M^{\prime} / 13$ and that the assertion has been proved for $j$. We shall proceed to establish it for $j+1$. Accordingly let there exist $j$ blocks $B_{p_{1}}, \ldots, B_{p_{j}}$ and $[j t / 2]$ pairs of integers lying outside these blocks but with differences lying in the neighbourhoods of these blocks. Denote the set consisting of the $[j t / 2]$ pairs by $S_{j}$. We let $B_{1}^{(j)}, \ldots$, $B_{5 j}^{(j)}$ include all the blocks each containing at least $t / 10$ elements of $S_{j}$. There can 
be at most $(11 t / 10) 6 j$ pairs with differences lying in the neighbourhoods of $B_{1}^{(j)}$, $\ldots, B_{5 j}^{(j)}, B_{p_{1}}, \ldots, B_{p_{j}}$, and at most $j t$ pairs lying in the blocks $B_{p_{1}}, \ldots, B_{p_{j}}$. Thus there are $\geqslant 19 M^{\prime} t / 20-76 j t / 10$ pairs lying outside $B_{p_{1}}, \ldots, B_{p_{j}}$ and whose differences lie outside the neighbourhoods of $B_{1}^{(j)}, \ldots, B_{5 j}^{(j)}, B_{p_{1}}, \ldots, B_{p_{j}}$. Hence there is one block say $B_{p_{j+1}}$ which contains the differences of at least $\left(19 M^{\prime} t / 20-76 j t / 60\right)\left(M^{\prime}-6 j\right)^{-1}$ pairs. In view of $j \leqslant M^{\prime} / 13$, this number is $\geqslant$ $t / 2+t / 5$. These pairs are clearly distinct from those in $S_{j}$. Furthermore $B_{p_{j+1}}$ contains at most $t / 10$ of the pairs just chosen (since $B_{p_{j+1}}$ is not a bad block) and at most $t / 10$ pairs from $S_{j}$ (since $B_{p_{j+1}}$ is different from $B_{1}^{(j)}, \ldots, B_{5 j}^{(j)}$ ). Hence we may now assert that there are $\geqslant[j t / 2]+(t / 2+t / 5)-t / 5 \geqslant$ $[(j+1) t / 2]$ pairs of integers lying outside $B_{p_{1}}, \ldots, B_{p_{j+1}}$ and whose differences lie in their neighbourhoods. This concludes the induction argument and with it the proof of Proposition P. This completes the proof of the lower bound in (1.1).

We remark that it is possible to iterate the process for the lower bound and obtain $f(n)>\sqrt{n} w^{2} / 2$ and by iterating $k$ times we may obtain $f(n)>\sqrt{n} w^{k} / k$ ! which grows up as high as $n^{1 / 2+\epsilon}$. As the proof of this involves rather messy details we have refrained from carrying this out in this paper. It is conceivable that $f(n)>$ $n^{1-\epsilon}$ for every $\epsilon$ and $n \geqslant n_{0}(\epsilon)$.

\section{REFERENCES}

1. P. Erdös, Extremal problems in number theory, Proc. Sympos. Pure Math., vol. 8, Amer. Math. Soc., Providence, R. I., 1965, pp. 181-189. MR 30 \#4740.

2. S. L. G. Choi, The largest sum-free subsequence from a sequence of $n$ numbers, Proc. Amer. Math. Soc. 39 (1973), 42-44. MR 47 \#1771.

3. D. Cantor (to appear).

4. S. L. G. Choi, On sequences not containing a large sum-free subsequence, Proc. Amer. Math. Soc. 41 (1973), 415-418. MR 48 \#3910.

5. V. Chvátal and J. Komlós, Some combinatorial theorems on monotonicity, Canad. Math. Bull. 14 (1971), $151-157$.

DEPARTMENT OF MATHEMATICS, UNIVERSITY OF BRITISH COLUMBIA, VANCOUVER, BRITISH COLUMBIA, CANADA HUNGARY

MATHEMATICS INSTITUTE, HUNGARIAN ACADEMY OF SCIENCES, BUDAPEST,

COMPUTER SCIENCE DEPARTMENT, STANFORD UNIVERSITY, STANFORD, CALIFORNIA 94305 